\title{
Inferring Volatility from the Yield Curve
}

\author{
Vincent Brousseau1, Alain Durré ${ }^{2}$ \\ ${ }^{1}$ https://www.researchgate.net/profile/Vincent Brousseau2 \\ ${ }^{2}$ IÉSEG-School of Management (Lille Catholic University) and LEM-CNRS, Lille, France \\ Email: a.durre@ieseg.fr
}

Received 21 July 2015; accepted 24 August 2015; published 28 August 2015

Copyright (C) 2015 by authors and Scientific Research Publishing Inc.

This work is licensed under the Creative Commons Attribution International License (CC BY).

http://creativecommons.org/licenses/by/4.0/

(c) (†) Open Access

\begin{abstract}
In this paper, we assess how to recover the volatility of interest rates in the euro area money market, on the sole basis of the zero-coupon yield curve. Our primary result is that there exists an empirical regularity (linking rates and volatility) that takes a relatively simple mathematical form. We also show that the existence of such regularity cannot be explained by a reasoning based on the hypothesis of absence of opportunities of arbitrage since a continuous-time arbitrage-free model may produce instances of curves that are consistent with a continuum of level of volatilities. We exhibit an example for this.
\end{abstract}

\section{Keywords}

\section{Yield Curve, Volatility, Consol Volatility, Affine Model}

\section{Introduction}

Estimating volatility of financial assets is essential to accurately assess the underlying risk and uncertainties attached to a particular investment. Recent developments in the sovereign debt market in the euro area (with skyrocketting volatility in the aftermath of the ECB asset purchase program) remind us of the importance of this parameter in asset pricing and hedging. Although long debated in the literature, various volatility models coexist and none seems to be dominant. However, the main non-model-based approach rooted in the paper [1], which was written by Litterman, Scheinkman and Weiss in 1991. In their paper, very interestingly, implicit volatility can be to a large extent recovered from the sole observation of the yield curve alone.

Similarly motivated as ours, their paper asserted that "understanding the effect of the interest rate volatility implicit in the yield curve is essential to comprehending the behavior of fixed-income securities". It presented a regression of the implied volatility from options on Treasury bond futures on the level of the 1-month, 3-year, and 10-year zero-coupon rates (from the Treasury curve). Their data sample covered four years and a half and contained weekly observations, so slightly less than 250 observation dates. They found a $R^{2}$ of $70 \%$. They 
concluded that the mere shape of the yield curve did contain a sizeable amount of information about implied volatilities of interest rates.

Two questions emerge naturally: Where could the $R^{2}$ originate from, and how could the result be refined?

The first question can be reformulated in terms of arbitrage-free model: can it be that the absence of arbitrage opportunities induces some relationship between the curve shape and its volatility, in a way that could be made apparent in a formalized arbitrage-free model of the curve's dynamics? The most positive way to answer this question would be to exhibit a fully-fledged arbitrage-free model such that no link can exist between the curve shape and its volatility. Ideally, it should be able to produce theoretical curves that are consistent with several risk-neutral distributions and several volatility levels. It turns out that such examples actually exist and we will present what is probably the simplest one in the continuous-time framework, and certainly the simplest one in the sub-class of affine models.

The second question aims at identifying the mathematical structure of the link existing between rates and volatility. This identification would have some market relevance since its knowledge would permit to construct trading strategies arbitraging the volatility against its value such as predicted by the curve. This second question falls into the category of pattern recognition or pattern indentification. Therefore, to adress it, it is necessary to rely on a very large data set. We will use a data sample with daily observations made on the euro money market. We will refine the specification of the exercise so as to eliminate as much as possible the dust of technical premia and of the aforementioned arbitrary choices. The curve shall be derived from overnight indexed swaps (OIS). The volatility shall be the consol volatility defined in [2], namely the instantaneous one of a perpetual bond (consol bond) priced on the curve, instead of having the positive and decaying times to maturity of a T-bond option or the 10-year maturity of its underlying asset. We will also give a simple expres- sion to the functional of the yield curve that replicates the volatility. That proxy achieves a somewhat better $R^{2}$ of $84 \%$, and, more importantly, it achieves it on 10 years of daily observations, which represent about ten times the number of observations of the original exercise of [1].

In summary, the proposed exercise highlights two elements of information. There is a recognizable empirical regularity linking the volatility to the shape of the curve, and this regularity cannot boil down to the absence of arbitrage opportunities.

The paper is organized as follows. In Section 2, we will briefly introduce the consol volatility indicator. In Section 3, we will show an example of affine model such that a given curve corresponds to infinitely many specifications of that model. In today's terminology, one says that the yield curve does not span interest rate volatility risk. The example we exhibit here is thought to be the simplest possible one in the frame of continuoustime affine models. We will show that such a curve is realistic and is coincident with the actual curve observed on the euro OIS market at a certain date. The curve is consistent with a large range of consol volatilities and we will calculate that range in the case of this observation date. Volatilities thus cannot in general be inferred from the shape of the curve if one relies on the hypothesis that the curve is generated by this affine model or by any more general model containing it as a subcase. In Section 4, we will exhibit an affine functional of the yield curve - which is of a-theoretical origin and thus is not justified by any arbitrage-free modeling argument—which is nevertheless a surprisingly good proxy of the consol volatility of the euro OIS curve. Volatilities thus may be inferred from the shape of the curve, although the volatility proxy may not have a justification grounded on arbitrage-free curve modeling. The reason that explains this empirical regularity linking shape and volatility has then to be researched outside the frame of arbitrage-free modeling, as the last section concludes.

\section{The Consol Volatility}

The volatility indicator that we will focus on is termed as the consol volatility. That indicator is extensively described in [2]; we only briefly introduce it here.

A consol bond is defined here as a perpetual bond paying continuously a constant rate of money, which is called the coupon flow. ${ }^{1}$ The consol price $\mathrm{C}$ is defined as the price of the consol bond divided by the coupon flow. The consol price is a function of the yield curve, which determinates it entirely. Indeed it can be expressed as follows:

${ }^{1}$ In the context of other pieces of literature, the consol bond is still defined as perpetual, but pays an annual or semi-annual coupon frequency instead of a continuous constant money flow. We choose to use the definition that is mathematically the simplest one. 


$$
C \doteqdot \int_{0}^{\infty} P(\theta) \mathrm{d} \theta
$$

where $P(\theta)$ is the price of a zero-coupon bond of residual maturity $\theta$. The consol volatility is the instantaneous Black and Scholes volatility of $C$. In what follows, we will skip the terms "instantaneous" and "Black and Scholes" and simply refer to it as to the consol volatility. As shown in [2], it is possible to recover the market-implied consol volatility of the OIS curve of the euro on the basis of various market data including, but not limited to, Euribor swaptions.

Contrarily to the T-bond options used in [1], neither consol rate nor consol volatility are directly traded, but both of them can be priced and hedged, thus synthetically traded-which amounts at saying that both of them are implied by actual market data. Our choice to focus specifically on the consol volatility is primarily justified by the fact that the consol bonds remains identical to themselves as time elapses, while actual bonds and swaps have a declining time to maturity (as they have a fixed date of maturity). Furthermore, consol indicators are free from arbitrary choices such as 10-year maturity or 6-month frequency of coupon/of repayment. Finally, when priced on the OIS curve, they are not affected by factors other than interest rate risk, such as liquidity risk, credit risk, redenomination risk, ${ }^{2}$ or usability as collateral.

It is also worth to emphasize the following: the ratio between an interest rate volatility and the duration of the instrument underlying that option can be approximated at first order by the ratio of the consol volatility and of the consol bond duration. Therefore, the consol volatility summarizes into one unique number a large part of the information contained in the complete market-implied volatility structure.

The question of the proposed exercise can then be reformulated in specific terms: "Can the consol volatility of the OIS curve be inferred from the shape of that curve?”

\section{Degenerate Case in an Affine Model}

In this section, we will show that it is possible to have a curve generated by an arbitrage-free model which could also have been generated by the same model with different parameters, and is consistent with several (different) risk-neutral distributions. We will furthermore show, yet only numerically, that it is consistent with a large range consol volatilities. The strategy for inferring the volatility from the curve shape, which consists into fitting it to an arbitrage-free model and then computing the volatility, will fail in such cases.

\subsection{The Mathematical Example of the Degeneracy}

The possibility that arbitrage-free affine models may contain situations where a given yield curve is consistent with more than one risk-neutral distribution is known since the early nineties. We read in [3], pp. 31-32, "cela entrane que [the variance covariance matrix and the drift vector] doivent être des fonctions affines de [the state vector] dès que cette équation peut être inversée. Ceci n'est plus garanti par l'hypotèse 1 et peut ne pas être vérifié dans certains cas non-génériques". ${ }^{3}$ To our knowledge however, the concrete example that we now present of such a degeneracy is the first to be known in the continuous-time framework. It is a particular case of the parabolic model of Gourieroux and Sufana [4], which is the generic case of the 2-factor affine model.

Following [2], the parabolic model can be rewritten as follows: There are two factors $r$ and $p, r$ being the short-term rate. The risk-neutral probability can be seen as the solution of the SDE:

$$
\left(\begin{array}{l}
\mathrm{d} r_{t} \\
\mathrm{~d} p_{t}
\end{array}\right)=\left(\left(\begin{array}{c}
a_{1} \\
a_{2}
\end{array}\right)-\left(\begin{array}{cc}
b_{11} & b_{12} \\
0 & b_{22}
\end{array}\right)\left(\begin{array}{l}
r_{t} \\
p_{t}
\end{array}\right)\right) \mathrm{d} t+\sqrt{\left(\begin{array}{cc}
c+r_{t} v^{2} & \frac{p_{t} v^{2}}{2} \\
\frac{p_{t} v^{2}}{2} & \frac{v^{2}}{4}
\end{array}\right)} \mathrm{d} \mathbf{W}_{t}
$$

with $\mathbf{W}_{t}$ a two-dimensional Wiener process. The short-term rate process $r_{t}$ evolves in $\left[r_{\min },+\infty[\right.$, with:

\footnotetext{
${ }^{2}$ In the case of the euro, the redenomination risk refers to the risk of being reimbursed in a currency that is no longer legal tender in Germany at the time of repayement. This is an important component of the spreads of government bonds to the German government curve or to the OIS curve.

${ }^{3}$ This reservation made by Frachot and Lesne (1995) may have been overseen in the sequel. It is not uncommon to find in the literature claims that the risk-neutral distribution of an affine model can always be determined by the curve.
} 


$$
r_{\min }=-\frac{c}{v^{2}}
$$

The second parameter process $p_{t}$ evolves in $\mathbb{R}$. The state vector $(r, p)$ evolves in the convex domain where the variance-covariance matrix is positive-semidefinite. This domain is delimited by a parabola, hence the name of the model. We shall exclude cases where the short term rate has a finite upper bond, as they are of low relevance. Parameters $a_{1}, a_{2}, b_{11}, b_{12}, b_{22}, c, v$ must then satisfy specific constraints, namely:

$$
\begin{aligned}
& v>0 \\
& b_{11}>0
\end{aligned}
$$

and either:

$$
\begin{aligned}
& 2 b_{22}-b_{11}>0 \\
& a_{1}>a_{1 \min }^{(0)}
\end{aligned}
$$

where:

$$
a_{1 \min }^{(0)}=b_{11} r_{\min }+\frac{v^{2}}{4}+\frac{\left(2 a_{2}+b_{12}\right)^{2}}{4\left(2 b_{22}-b_{11}\right)}
$$

or:

$$
\begin{aligned}
& 2 b_{22}-b_{11}=0 \\
& b_{12}=-2 a_{2} \\
& a>a_{1 \min }^{(1)}
\end{aligned}
$$

where:

$$
a_{1 \min }^{(1)}=b_{11} r_{\min }+\frac{v^{2}}{4}
$$

It will be convenient to introduce two auxiliary parameters $\gamma$ and $\epsilon$ :

$$
\begin{aligned}
& \gamma \doteqdot \sqrt{b_{11}^{2}+2 v^{2}} \\
& \epsilon \doteqdot 2 b_{22}-b_{11}
\end{aligned}
$$

Note that the conditions (5) $\vee(7)$ imply that $\gamma>0$ and $\epsilon \geq 0$. Furthermore, since $\epsilon=2 b_{22}-b_{11}$, those conditions reduce either to (5) if $\epsilon>0$, or to (7) if $\epsilon=0$.

The zero-coupon bond price of maturity $t$ takes the form of the expectation:

$$
P(t, r, p)=\mathbf{E}\left[\mathrm{e}^{-\int_{0}^{t} r_{s} \mathrm{~d} s}\right] \mid r_{0}=r, p_{0}=p
$$

where the expectation $\mathbf{E}$ is taken under the risk-neutral probability (2). Therefore the zero-coupon bond price depends of the seven parameters $a_{1}, a_{2}, b_{11}, b_{12}, b_{22}, c, v$, of the two variables $r$ and $p$, and does not depend explicitly on time.

Since $P$ has the form (10), the model is arbitrage-free. The drift and the variance-covariance matrix in (2) are affine functions of the state vector $(r, p)$. Thus for any fixed $t>0, \log (P(t, r, p))$ is an affine function of the state vector $(r, p)$. Then the model is by definition affine.

We make the hypothesis:

$$
\epsilon=3 \gamma
$$

That hypothesis implies that $\epsilon>0$, since one has always $\gamma>0$.

We introduce a continuous group of transformations $T$ acting on the 9-tuple $\left(r, p, a_{1}, a_{2}, b_{11}, b_{12}, b_{22}, c, v\right)$, indexed by a parameter $h \in \mathbb{R}$, as follows:

$$
\begin{aligned}
& T(h)\left(r, p, a_{1}, a_{2}, b_{11}, b_{12}, b_{22}, c, v\right) \\
& =\left(r(h), p(h), a_{1}(h), a_{2}(h), b_{11}(h), b_{12}(h), b_{22}(h), c(h), v(h)\right)
\end{aligned}
$$


where:

$$
\begin{array}{ll}
r(h) & =r \\
p(h) & =p+3 h\left(b_{22}-2 b_{11}\right) \\
a_{1}(h) & =a_{1}+3 h b_{12}\left(b_{22}-2 b_{11}\right) \\
a_{2}(h) & =a_{2}+h\left(b_{22}-2 b_{11}\right)\left(2 b_{22}-b_{11}\right) \\
b_{11}(h) & =b_{11} \\
b_{12}(h) & =b_{12} \\
b_{22}(h) & =b_{22} \\
c(h) & =c+h b_{12}\left(b_{22}-2 b_{11}\right)\left(b_{11}+b_{22}\right) \\
v(h) & =v
\end{array}
$$

So $T(0)$ is the identity. We denote with $M(h)$ the transformed model in which each parameter or variable has been replaced by its transformation under $T(h)$. The parameters of the original model $M=M(0)$ satisfy (5) since $\epsilon>0$. Thus, by continuity, there exists some bound $H>0$ such that, for $|h|<H$, the model $M(h)$ 's parameters also satisfy (5).

We denote with $R(h)$ the probability law of the process $r_{t}$ for the risk-neutral distribution of the model $M(h)$. We denote with $P^{h}$ the zero-coupon bond price of the model $M(h)$.

Lemma 1 Assuming (5), $\epsilon=3 \gamma$ and $b_{12} \neq 0$, for any $h_{1}, h_{2}$ such that $M\left(h_{1}\right)$ and $M\left(h_{2}\right)$ 's parameters satisfy (5) and $h_{1} \neq h_{2}$, one has $R\left(h_{1}\right) \neq R\left(h_{2}\right)$.

The proof can be found in the Annex.

This lemma ensures that the transformation $T$ truly affects the risk-neutral law of the short-term rate unless $b_{12}$ is zero.

Lemma 2 Assuming (5), $\epsilon=3 \gamma$ and $b_{12} \neq 0$, for any $h_{1}, h_{2}$ such that $M\left(h_{1}\right)$ and $M\left(h_{2}\right)$ 's parameters satisfy (5) and $h_{1} \neq h_{2}$, for any $t \geq 0, P^{h_{1}}\left(t, r\left(h_{1}\right), p\left(h_{1}\right)\right)=P^{h_{2}}\left(t, r\left(h_{2}\right), p\left(h_{2}\right)\right)$.

The proof can be found in the Annex.

This lemma ensures that the transformation $T$ does not affect the yield curve.

The two lemmas yield the following:

Theorem Assuming (5), $\epsilon=3 \gamma$ and $b_{12} \neq 0$, the yield curve is consistent with infinitely many specifications of the parabolic model.

The proof can be found in the Annex.

\subsection{Numerical Examination with Actual Market Data}

The degenerate case is realistic. We pick up in our data sample an example of the OIS curve that is well-fitted by a parabolic model curve fullfilling the hypothesis of the Theorem, so corresponding to not only one 9-tuple $\left(r, p, a_{1}, a_{2}, b_{11}, b_{12}, b_{22}, c, v\right)$ of state variables and parameters, but to a continuum of those and to infinitely many risk-neutral distributions. In other terms, the curve does not span the interest rate volatility risk. The following Figure 1 depicts the actual curve together with the model curve.

Define the goodness-of-fit as the $L^{2}$ norm of the difference of the zero-coupon rates of the two curves between maturity 0 and 20-year. We compute it and find it to be equal to 2 basis points.

As the fitting curve is consistent with infinitely many specifications of the model, it might be consistent with a continuum of consol volatilities. For $h$ describing the set of admissible values such that $M(h)$ satisfies the conditions (5), we compute those volatilities and find that they range from $4.7 \%$ to slightly more than $250 \%$. The consol volatility actually implied by the market on that day was $16.5 \%$. That value falls within the range, yet that range is too large to convey any information.

\section{Proxying the Volatility from the Shape of the Curve}

In this section, we will show that the shape of an actual curve, observed in the market, nevertheless contains a considerable amount of information about the volatility. Working with the euro OIS curve, we will present a proxy constructed uniquely from the curve that closely matches the consol volatility. 


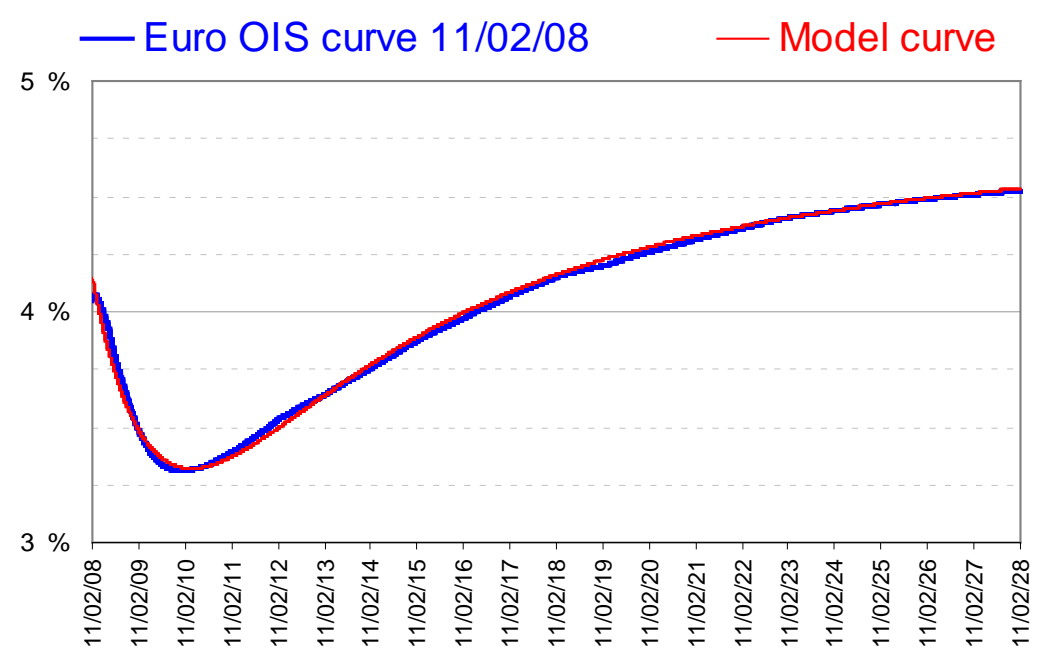

Figure 1. Euro OIS curve on 11 February 2008 (bold line), model curve (thin line) (zero-coupon rates, continuously compounded, day count act/365).

\subsection{Data}

The data sample is the same as in [2], covering all 3816 TARGET days from the start of the euro to 21 November 2013. It includes all the instruments needed for obtaining the euro OIS curve and the market-implied consol volatility. For a detailed description of the bootstrapping of the curve, we refer to [5], ${ }^{4}[6] .{ }^{5}$ For a detailed description of the reconstruction of the consol volatility, we refer to [2]. ${ }^{6}$

\subsection{The Proxy}

The proxy of the logarithm of the consol volatility is defined as an affine function of the curve. Denote with $P$ the zero-coupon price, with $f$ the instantaneous forward rate:

$$
f \doteqdot-\frac{\partial_{t} P}{P}
$$

Denote with $\sigma$ the consol volatility and with $\lambda$ the proxy of $\log (\sigma)$. We will look for a proxy depending of the function $f(\cdot)$ in an affine manner (it would have been equivalent to replace the forward rate by the zero-coupon rate or by the log zero-coupon price, since the conversion between any two of those three functions is linear).

Let us first briefly explain how we could get a specific expression for such a proxy. To guess what could be the form of the proxy, we rely on the method developed in [6] which will allow to express the function $f(\cdot)$ in an affine manner in function of a small number of factors. For the curve under study, the euro OIS curve, [6] finds that 7 factors are sufficient to limit the loss of accuracy to the typical size of the bid-ask spread (to only 1 basis point for maturities at or above 2-month, and only slightly larger for short-term maturities). One can then regress $\log (\sigma)$ on those factors. As the factor loadings of those 7 factors are known functions of the time to maturity, the regression leads to an expression of $\log (\sigma)$ of the form:

$$
\log (\sigma) \simeq \int_{T_{0}}^{T_{1}}\left(F_{0}(t)+F_{1}(t) f(t)\right) \mathrm{d} t
$$

with $0 \leq T_{0}<T_{1}<+\infty$ and $F_{0}(\cdot)$ and $F_{1}(\cdot)$ are functions of the time to maturity only. We are looking here for an a-theoretical proxy, not grounded on an underlying model and in particular on an arbitrage-free model of the form (15), so the last step is to try and recognize some simple mathematical expression in $F_{0}(\cdot)$ and $F_{1}(\cdot)$, which at this stage are known only as purely numerical data.

It turns out that this recognition is easy and we obtain therefore the mathematical shape of our proxy $\lambda$ as

${ }^{4}$ See Brousseau (2002) § 3.2.2. pp. 21-22.

${ }^{5}$ See Brousseau and Durré (2014) § 4.2.3. pp. 302-303.

${ }^{6}$ See Brousseau and Durré (2015) Annex, § 2.2. pp. 70-72. 
follows:

$$
\lambda=\alpha+\beta \int_{0}^{T_{1}} \cos (2 \log (t)) t^{-\frac{3}{4}} f(t) \mathrm{d} t
$$

still with $T_{1}<+\infty$. Observe that the convergence of the integral would be problematic if $T_{1}$ was $+\infty^{7}$ (This is a symptom that a proxy of that form cannot be derived from continuous-time curve modeling). We will then fix the value of $T_{1}$ to 30-year, because the longest OIS present in the whole sample is 30-year. Linear regression over the 10 last years of the available sample of $\sigma$ on the integral in (16) will then determine the remaining constants $\alpha$ and $\beta$.

Regarding those two constants, one should be attentive to the fact that both rates of volatilities are frequently expressed as percentages rather than as pure numbers. If the forward rate $f$ in input is expressed as percentage rather than as pure number, the constant $\beta$ should be divided by 100; if the volatility $\sigma$ in output is expressed as percentage rather than as pure number, the constant $\alpha$ should be augmented by $\log (100)$. To avoid confusion, the following Table 1 reports the values of constants $\alpha$ and $\beta$ depending of the four possible choices of either pure number or percentage for the forward $f$ and the volatility $\sigma$.

It turns out that the proxy works well only over the 10 last years of the available sample. For prior years, neither the proxy nor any other function of the yield curve only seems to be able to reproduce the volatility with a comparable explanatory power, or at least, our attempts to find one have failed. For the 10 last years, however, which represent a subsample covering 2565 observations, the proxy does a surprisingly good job.

The following Figure 2 depicts the logarithm of the actual market-implied consol volatility together with its proxy.

Table 1. Values of constants $\alpha$ and $\beta$.

\begin{tabular}{cccc}
\hline Forward rate & Volatility & $\alpha$ & $\beta$ \\
as pure number & as pure number & $-57.680,785,09$ & $4.214,443,943$ \\
as percentage & as pure number & $-57.680,785,09$ & $0.042,144,439$ \\
as pure number & as percentage & $-53.075,614,9$ & $4.214,443,943$ \\
as percentage & as percentage & $-53.075,614,9$ & $0.042,144,439$ \\
\hline
\end{tabular}

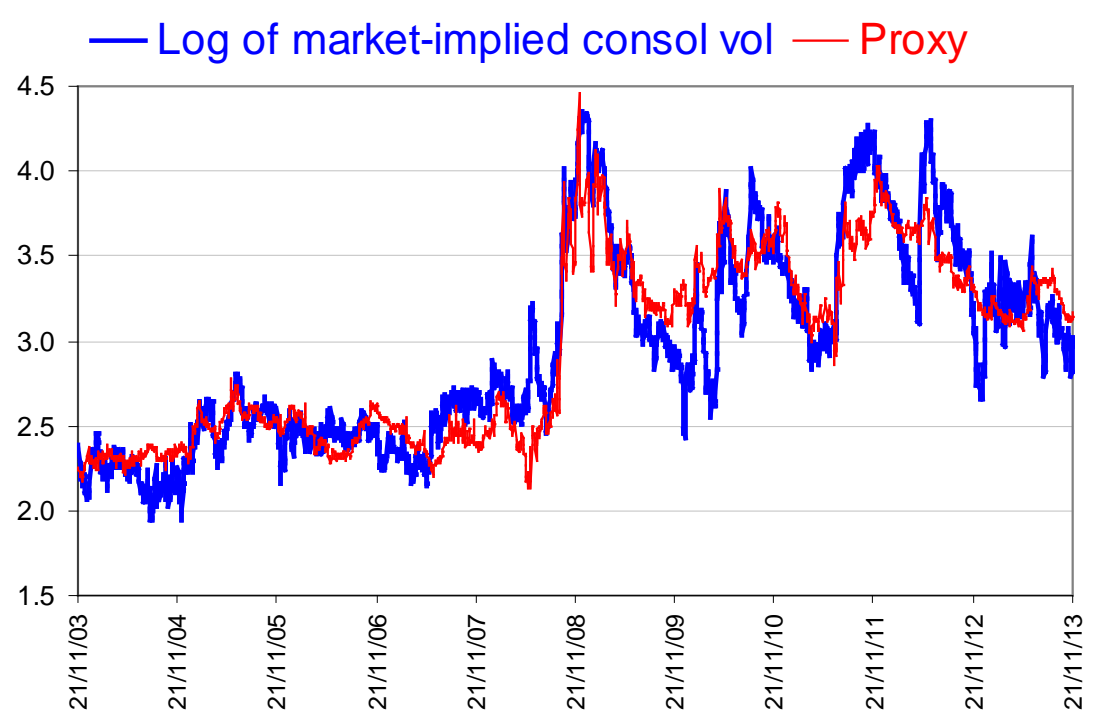

Figure 2. Log of the actual consol vol (bold line), proxy (thin line).

\footnotetext{
${ }^{7}$ Convergence of the integral in 0 is ensured since

$\int_{0}^{T_{1}} \cos (2 \log (t)) t^{-\frac{3}{4}} f(t) \mathrm{d} t=\frac{4}{65} \sqrt[4]{T_{1}}\left(\cos \left(2 \log \left(T_{1}\right)\right)+8 \sin \left(2 \log \left(T_{1}\right)\right)\right)$
} 


\subsection{Statistical Properties}

By computing the correlation and $R^{2}$ of the log volatility and its proxy, we find:

$$
\begin{aligned}
& \text { Corr }=91.7 \% \\
& R^{2}=84.2 \%
\end{aligned}
$$

The standard deviation of the error $\lambda-\log (\sigma)$ is:

$$
\text { StDev }=0.232
$$

which means that the proxying will typically overestimate or underestimate the consol volatility by a factor of $4 / 5$ or $5 / 4$. This is remarkable if one take into account that the proxy relies only on the shape of the curve and does not contain any market volatility data, but also that over the 10 years of the subsample, the behavior of the volatility has been extremely hectic.

\subsection{Comparison with the Fitting of the Curve to an Affine Model}

Let us consider the case of the example seen above in Section 3.2. The observation date was 11 February 2008. The consol volatility actually implied by the market on that day was $16.5 \%$. Fitting the curve on the 2-factor affine model could be achieved with a high accuracy, but the model curve was consistent with a large range of volatilities from $4.7 \%$ to slightly more than $250 \%$. The computation of the proxy from the actual curve leads to a value of $14.8 \%$. While both methods rely on the sole knowledge of the curve, it is clear that the second one obtains better results.

\subsection{Practical Implications: Trading Strategies}

The error, or difference between the log vol and its proxy, exhibits a strong mean reversion. This opens the way for building trading strategies. We will not enter here into a detailed description, but we will briefly explain the principle.

The curve motion is empirically dominated by parallel shifts, as this is well-known since [7]. Therefore, one can approximate the volatility (even the non-instantaneous volatility) of an interest rate instrument by the consol volatility multiplied by the duration of the instrument and divided by the consol duration. It follows that any interest rate option can be deemed either cheap or rich with respect to the actual state of the OIS curve, just by considering the sign of the error. That sign of the error is observable in real time.

For instance, if the proxy is lower than the actual log volatility, then one should expect a convergence of the two figures towards each other. One should then sell the volatility and enter in a portfolio of swap rates constructed so as to be sensitive to that combination of interest rates defined in (16) and hedged against other components of the curve's motion. The construction of such a portfolio rate relies of course on a principal component analysis of the curve motion. The relative size of the option selling position and of the portfolio of swaps is then determined by the vega of the option and by the sensitivity of the portfolio to the proxy.

\section{Concluding Remarks}

This article has examined the role of the shape of the yield curve in determining interest rate volatility. It focuses on a particular volatility indicator called consol volatility. The sole hypothesis of arbitrage-freeness cannot explain the existence of such a connection. In effect, there exist arbitrage-free models where a given curve shape is consistent with a continuum of volatility levels, and we gIve an example drawn from the category of continuoustime affine models. Nevertheless, the shape of the zero-coupon curve of OIS for the euro is shown to contain a substantial amount of information about the volatility level, and we have shown how to recover it by constructing explicitly a proxy of the consol volatility from the curve only,.

\section{References}

[1] Litterman, R., Scheinkman, J. and Weiss, L. (1991) Volatility and the Yield Curve. Journal of Fixed Income, 1, 49-53. http://dx.doi.org/10.3905/jfi.1991.692346

[2] Brousseau, V. and Durré, A. (2015) Interest Rate Volatility: A Consol Rate Approach. Journal of Mathematical Finance, 5, 58-72. http://dx.doi.org/10.4236/jmf.2015.51006 
[3] Frachot, A. and Lesne, J.-P. (1995) Modèles factoriels de la structure par termes des taux d'intérêt: Théorie et application économétrique. Annales d'Économie et de Statistique, 40, 11-36.

[4] Gourieroux, C. and Sufana, R. (2006) A Classification of Two-Factor Affine Diffusion Term Structure Models. Journal of Financial Econometrics, 4, 31-52. http://dx.doi.org/10.1093/jifinec/nbj003

[5] Brousseau, V. (2002) The functional Form of Yield Curves. ECB Working Paper No. 148. https://www.ecb.europa.eu/pub/pdf/scpwps/ecbwp148.pdf

[6] Brousseau, V. and Durré, A. (2014) Sur une représentation paramétrique affine de la courbe des taux. Revue Bancaire et Financière, 4, 297-310.

[7] Litterman, R. and Scheinkman, J. (1991) Common Factors Affecting Bond Returns. The Journal of Fixed Income, 1, 54-61. http://dx.doi.org/10.3905/jfi.1991.692347 


\section{Annex}

\section{Proofs of Lemmas and Theorem}

\subsection{Proof of Lemma 1}

Let $T>0$. The set of all trajectories of $r$ is $C([0, T])$, the set of continuous functions from $[0, T]$ to $\mathbb{R}$, and it is endowed with its Borel $\sigma$-algebra. A probability law of $r_{t}$ is a measure on it which is positive and sums up to 1. Denote with $D(h)$ the set of functions $f$ of $t$ such that the quadratic variation of $f$ exists for any $t \geq 0$, is differentiable for any $t \geq 0$, and its derivative is $c(h)+f(t) v^{2}$ for any $t \geq 0$.

$$
D(h) \doteqdot\left\{f \in C([0, T]) / \forall t \in[0, T],\langle f\rangle(t)<+\infty, \partial_{t}\langle f\rangle(t)=c(h)+f(t) v^{2}\right\}
$$

If $\epsilon=3 \gamma$ the expression (13) of the transformation of $c$ reduces to:

$$
c(h)=c+\frac{9}{2} h b_{12} v^{2}
$$

so, as $h_{1} \neq h_{2}$ and $b_{12} \neq 0$, one has $c\left(h_{1}\right) \neq c\left(h_{2}\right) . D(h)$ is a measurable set. The measure of $D(h)$ under $R(h)$ is 1 if $h_{1}=h_{2}, 0$ if $h_{1} \neq h_{2}$. Hence, if $h_{1} \neq h_{2}$, then $R\left(h_{1}\right) \neq R\left(h_{2}\right)$.

\subsection{Proof of Lemma 2}

Let $U(t, r, p)=-\log (P(t, r, p)), U^{h}(t, r, p)=-\log \left(P^{h}(t, r, p)\right)$. Applying Feynman-Kac formula to (10) and then expressing the $P$ as $\exp (-U)$ yields that $U$ satisfies the PDE:

$$
\begin{aligned}
& \frac{1}{2}\left(\begin{array}{ll}
\partial_{r} U & \partial_{p} U
\end{array}\right)\left(\begin{array}{cc}
c+r v^{2} & \frac{p v^{2}}{2} \\
\frac{p v^{2}}{2} & \frac{v^{2}}{4}
\end{array}\right)\left(\begin{array}{l}
\partial_{r} U \\
\partial_{p} U
\end{array}\right) \\
& -\left(\left(\begin{array}{l}
a_{1} \\
a_{2}
\end{array}\right)-\left(\begin{array}{cc}
b_{11} & b_{12} \\
0 & b_{22}
\end{array}\right)\left(\begin{array}{l}
r \\
p
\end{array}\right)\right) \cdot\left(\begin{array}{l}
\partial_{r} U \\
\partial_{p} U
\end{array}\right)+\partial_{t} U-r=0
\end{aligned}
$$

with the limit condition:

$$
U(0, r, p) \equiv 0
$$

and conversely, if $U$ satisfies (21)-(22), then $P=\exp (-U)$ is the zero-coupon price of the model $M$.

The proof of the lemma will not require us to solve explicitly (21)-(22). Instead, we just have to find the expressions of $\left(\partial_{r} U, \partial_{p} U\right)$. Recall that the model is affine, so $\partial_{r} U$ and $\partial_{p} U$ are functions of $t$ only, and not of $(r, p)$. Differentiating (21) w.r.t. $(r, p)$ yields:

$$
\begin{aligned}
& -1+b_{11} \partial_{r} U+\frac{1}{2} v^{2}\left(\partial_{r} U\right)^{2}+\partial_{t}\left(\partial_{r} U\right)=0 \\
& b_{12} \partial_{r} U+\partial_{p} U\left(b_{22}+\frac{1}{2} v^{2} \partial_{r} U\right)+\partial_{t}\left(\partial_{p} U\right)=0
\end{aligned}
$$

Those are standard Ricatti equations whose solutions are found directly for the first one, and for the second one after having reported in it the solution of the first one.

$$
\begin{aligned}
& \partial_{r} U=2 \frac{1-\exp (-\gamma t)}{\left(\gamma+b_{11}\right)+\left(\gamma-b_{11}\right) \exp (-\gamma t)} \\
& \partial_{p} U=-4 b_{12} \frac{\frac{1}{\gamma+\epsilon}+\frac{2 \gamma}{(\epsilon+\gamma)(\epsilon-\gamma)} \exp \left(-\frac{\gamma+\epsilon}{2} t\right)+\frac{1}{\gamma+\epsilon} \exp (-\gamma t)}{\left(\gamma+b_{11}\right)+\left(\gamma-b_{11}\right) \exp (-\gamma t)}
\end{aligned}
$$

At this point, we make use of the hypothesis $\epsilon=3 \gamma \cdot \partial_{p} U$ simplifies to

$$
\partial_{p} U=-b_{12} \frac{1-\exp (-\gamma t)}{2 \gamma} \partial_{r} U
$$


Thus $\left(\partial_{r} U, \partial_{p} U\right)$ also satisfies a second PDE:

$$
\frac{1}{2}\left(\begin{array}{ll}
\partial_{r} U & \partial_{p} U
\end{array}\right)\left(\begin{array}{cc}
b_{12} & \frac{1}{2}\left(\gamma-b_{11}\right) \\
\frac{1}{2}\left(\gamma-b_{11}\right) & 0
\end{array}\right)\left(\begin{array}{c}
\partial_{r} U \\
\partial_{p} U
\end{array}\right)-\left(\begin{array}{c}
0 \\
-1
\end{array}\right) \cdot\left(\begin{array}{c}
\partial_{r} U \\
\partial_{p} U
\end{array}\right)=0
$$

Multiplying (26) by $-\left(2 b_{11}-b_{22}\right)\left(b_{11}+b_{22}\right) h$ and adding the result term to (21) yields a third PDE. One recognizes in it the original (21) in which the seven parameters $a_{1}, a_{2}, b_{11}, b_{12}, b_{22}, c, v$ have been substituted by $a_{1}(h), a_{2}(h), b_{11}(h), b_{12}(h), b_{22}(h), c(h), v(h)$ such as given in (13). As a consequence, $P=\exp (-U)$ is also the zero-coupon price of the model $M(h)$, which concludes the proof.

\subsection{Proof of Theorem}

For $|h|<H$, the application $h \rightarrow M(h)$ is injective by Lemma 1, hence the set $M([-H, H])$ is infinite. 